ON THE

\title{
DIAGNOSIS AND TREATMENT OF RUPTURED BLADDER.
}

\author{
BY \\ CHRISTOPHER HEATH, F.R.C.S., \\ HOLME PROFESSOR OF CLINICAI SURGERY IN UNIVERSITY COLLEGE, LONDON,
} AND SURGEON TO UNIVERSITY COLLEGE HOSPITAL.

(Receired December 10th, 1878-Read February 25th, 1879.)

Having recently had under my care a case of rupture of the bladder into the peritoneum, in which $I$ opened the abdomen and closed the aperture in the bladder with stitches, with a fatal result, I have been led to look closely into the history of recorded cases of ruptured bladder with the following results.

The symptoms of ruptured bladder are often not so marked as might be expected from the classical descriptions of textbooks. A man with a full bladder receiving a blow upon the belly, may no doubt be conscious of something giving way and become faint; but where a severe injury, such as a "buffer accident," has been inflicted upon the pelvis, it is not uncommon to find, post-mortem, a rupture of the bladder which has given rise to no special abdominal symptoms during the last few hours of life. Indeed, so equivocal often are the symptoms that the reputed cases of recovery after rupture of the bladder have formed grounds for disputation between able surgeons as to whether or not the viscus was really ruptured. 
In my own case the patient was under medical care for an entire day without the accident being recognised, and on admission to the hospital his case was regarded as one of injury to the kidney rather than the bladder, until my visit a few hours later. Indeed, had my patient recovered without operative interference, $I$ believe he might have been said to have escaped injury to the bladder, as in Mr. Chaldecott's and Dr. Thorp's cases.

For the following detailed report of my case I am indebted to Mr. Samuel Burton, late Surgical Registrar of University College Hospital.

Jesse B-, æt. 47, a labourer, was admitted into University College Hospital, 11 a.m., November 25th, 1878, with the following history:-At 9.30 p.m., November 23rd, after patient had drunk two pints of beer, he began "larking about" with his companions, one of whom threw him violently on his back by putting his leg behind patient and forcing him backwards by his elbow, which hit him "in the stomach." He suffered great pain after the fall, and was taken home, where he was attended by a doctor who sent the following account with him:- "Patient is suffering from hæmaturia and retention of urine. A No. 6 catheter has been passed at intervals, but only a few ounces of bloody urine have been drawn off, the bladder remaining distended. He has been catheterised this morning (November 25th) with the same result as before. Opium has been given since November 24th, a.m., and patient appears better." Patient is quite sure he passed water freely half an hour before the accident.

Condition on admission.-Patient looks very ill, is extremely anæmic, has an anxious expression of countenance, is breathing quickly, mainly thoracic. Skin is moist. Pulse feeble and rapid. Pupils contracted from opium. Patient is lying on his right side with his knees drawn up, complaining of great pain in the lower part of the belly.

Examination of abdomen.-The skin over the front of the abdomen is reddened by a sinapism. The abdomen is pro- 
minent in front, no bulging of the flanks ; it is firm and tense to the feel, and very painful on the slightest pressure, notably over the hypogastrium. There is dulness above the pubes to a point midway between pubes and umbilicus. The left flank is dull on percussion, the right resonant; on turning patient over to right side, the left flank becomes resonant. There is no bruising of the back, but there is tenderness in the left lumbar region.

A No. 7 catheter was passed (this was the largest size that could be passed, owing to a stricture three inches from meatus) and three ounces of bloody urine drawn off, which came in an intermittent stream. No more urine coming away, the catheter was withdrawn, and the eyes were found to be plugged by a clot of blood. There is no pain on pressure over perinæum, and the pubic symphysis and arches are intact.

At his visit Mr. Heath saw the patient at 3 p.m., and diagnosed ruptured bladder. Patient having consented to undergo an operation he was taken into the theatre at 4 p.m., and ether was administered.

Mr. Heath first of all passed a No. 7 catheter, and found that bloody urine was ejected by intermittent, irregular jerks, corresponding to expiration. About half a pint was withdrawn. The catheter passed a long way, but was not felt to go through any rent in the bladder; it was freely movable, as if in some large cavity. The pubes being shaved and washed with carbolic lotion, an incision was made in the middle line just above the pubes for two inches, and the tis. sues divided down to the peritoneum, which appeared blue, the recti muscles which were firmly contracted being held aside by retractors with difficulty. The peritoneum was then picked up and a cut made into it, when a gush of fluid like that drawn off by the catheter came out; a large quantity of clots was then taken out from the peritoneal cavity. Mr. Heath having introduced his finger into the peritoneal cavity, found a long rent in the posterior wall of the bladder high up. It was proved to be a rent in the bladder, by passing a catheter through it from the urethra. The rent was then sewn up by 
a continuous catgut suture firmly tied at both ends. The clots were removed as far as possible from the peritoneum and the cavity sponged out after injection with warm water, and a long large-sized drainage-tube was inserted at the lower angle of the wound, the upper part of the wound being brought together by deep and superficial sutures. The carbolic spray ceased working before the operation was completed. A catheter was passed into the bladder, to which was afterwards attached some india rubber tubing leading into a vessel under the bed. Patient being put back to bed, a hot poultice was applied to the abdomen, and opium was administered, gr. $\mathrm{j}$ in pil. 4 tis horis.

At 11 p.m. patient expressed himself as much better. His anxious Hippocratic aspect had passed off; pulse had improved; no sickness; abdominal pain much less ; distension relieved.

November 26th, 10 a.m.-Patient has passed a quiet night, almost free from pain. He lies with legs outstretched.

Urine.-The amount that has drained away since the operation is $28 \frac{1}{2}$ ounces; it is apparently free from blood, acid, sp. gr. 1018, contains one-twelfth albumen, colour brownish-yellow, somewhat turbid; under the microscope some red blood discs are seen. Temp. $99^{\circ} 2^{\circ}$; pulse 112 , moderately full, and less compressible.

Examination of abdomen.-The prominence and distension are much less marked, and there is but little pain on pressure. The bowels have not acted, but flatus has been passed per anum. The drainage-tube was removed, a little bloody fluid being expressed from the wound. Poultices continued. He has taken three eggs and brandy 3 ij every hour.

November 27th, 10 a.m.-Patient has passed a quiet night, sleeping for the greater part of the time. He is dozing now, and lies with legs outstretched. Temp. $98 \cdot 2^{\circ}$; pulse 112 ; no sickness. Since 9 p.m. last night patient has passed four copious stools.

Urine. $-44 \frac{1}{2}$ ounces passed in the last twenty-four hours, 
besides some into the bed, of normal colour, acid, and free from blood.

The abdomen is a little more distended, but not painful, except at the epigastrium. There is but little discharge from the wound, only sufficient to soak a small piece of oakum placed over the wound, and which is changed every four hours.

November 28th, 10 a.m.-Patient has slept well through the night, complains of feeling "blown up with wind." No sickness or hiccough. Tongue moist, coated with a thick, yellow fur. Temp. $98^{\circ}$; pulse 100 . The abdomen is very tense but not painful, the left flank is dull. A considerable quantity of fluid has drained from the wound, the edges of which are sloughy.

Urine. -92 ounces passed in the last twenty-four hours, all but the last four ounces of normal colour, contains no albumen, and is free from blood. These four ounces are of a bright red colour, slightly acid. Sp. gr. 1020, one-twelfth albumen.

November 29th, 10 a.m.-Patient passed a very restless night. He has had constant vomiting and can keep nothing down. Has passed several motions into the bed. He is looking very ill this morning, expression anxious, and features pinched. Pulse small and indistinct; temp. 98.4.

Abdomen is very distended and somewhat painful. The wound looks very sloughy, and there has been scarcely any discharge of sero-purulent fluid.

Urine. $-45 \frac{1}{2}$ ounces passed of a chocolate colour, nearly solid, with albumen; neutral. Sp. gr. 1018.

4 p.m.-Patient is much worse. Sickness and diarrhœa continue.

10 p.m.-Patient is sinking fast.

11 p.m.-Died, six days after the accident.

November 30th.-Post-mortem made at 2 p.m., fifteen hours after death, weather cold, rigor mortis well marked.

The abdomen being opened by a crucial incision, the small intestines were seen considerably distended. For two inches around the wound in the abdominal wall the intestines were adherent by recent lymph to each other, and to the abdo- 
minal parietes. Above and on each side of these adhesions there was no trace of peritonitis. On tearing away these adhesions, some coils of intestine were seen lying over the pelvis, glued together and to adjacent parts by recent bloodstained lymph. On lifting these coils upwards, the rectovesical pouch of peritoneum was exposed, containing about six ounces of clotted blood, black in colour, and moderately offensive in odour. The rent in the bladder was also visible, the lower part of the wound gaping. Some blood lay over the cæcum and the ascending colon; this had probably overflowed from the pelvis.

The thorax was then opened. Heart and lungs apparently quite healthy. Iiver large and fatty, weight 4 pounds 2 ounces. Spleen normal. Intestines removed, no sign of enteritis along the whole tract.

Kidneys and pelvic organs removed en masse. There is no sign of pelvic cellulitis or of extra-peritoneal hæmorrhage. No injury to the large vessels of the abdomen or pelvis. No fracture of the symphysis pubis, or any of the pelvic bones.

Examination af bladder.-There is a rent in the mid-line of the posterior wall of the bladder 2 inches in length, extending upwards as high as the apex. The lower third of the rent was gaping, the edges of the rest were approximated by the catgut suture, the lower end of which was free and loose. The bladder was slit up in the middle line in front, and on each side close to the prostate ; the mucous membrane was blood-stained.

The recto-vesical pouch was lined by tolerably firm, blood-stained, adherent lymph.

Kidneys are of normal size, capsules smooth and strip off readily. Pelves and ureters healthy.

Brain and its membranes healthy.

My reasons for believing that the bladder was ruptured were :- 1 , the pallid pinched appearance of the face betokening some serious visceral lesion; 2 , the distended condition of the abdomen; 3 , the fact that a small quantity of clear urine was drawn by the catheter immediately on reach- 
ing the bladder, and on further introduction a quantity of bloody fluid presumably from the peritoneal cavity; 4, that warm water injected through the catheter was distinctly felt by the patient in the groins and abdomen. The passage of fluid from the peritoneal cavity became more apparent when the patient was breathing heavily under ether, for then the fluid flowed and stopped with each respiration. This symptom was noticed also by Mr. Willett in his case, 'St. Bartholomew Hospital Reports,' 1876, and I am inclined to lay stress upon it, since so long as the muscular coats of the bladder are entire, $I$ believe micturition never is so directly influenced by the respiration.

My reasons for selecting the proceeding I did were, 1 , the hopelessness of the case if left untreated; 2 , the fact that the operation had been recommended by Mr. Holmes in his 'Principles and Practice of Surgery,' although I was not aware of its having been performed. I have since found that Mr. Willett performed the operation at St. Bartholomew's Hospital in 1876, using interrupted silk sutures, the patient dying the next day. Mr. Willett's case in many respects resembled mine, but a somewhat lesser time had elapsed between the accident and the operation. I can fully confirm Mr. Willett's remarks as to the difficulty of the operation, due to the muscular condition of the abdominal walls; but I used a smaller incision than he did, and consequently did not allow of any escape of the intestines.

I must confess my surprise at the very large quantity of blood effused into the peritoneum by a rupture of a not highly vascular organ such as the bladder. On opening the abdomen I removed a quantity of clot, and subsequently washed out more, but after death a considerable quantity was found in the recto-vesical pouch. Rather against my own judgment, at the time of the operation $I$ introduced a large india-rubber perforated drainage-tube into the pelvis, and secured it at the lower end of the wound, and I find that Mr. Willett adopted the same practice. Finding that nothing came through the tube, but that clear serous fluid from the peritoneum welled out by the side, I withdrew it on the 
second day, and I cannot but think that its presence was harmful to the patient. The peritonitis was limited to the pelvis, and its greatest intensity had been exactly where the tube passed. Had I introduced a tube through the rectovesical pouch of peritoneum and brought it out at the anus I should have established a dependent opening, and been able to wash out the blood which collected there. Tapping the recto-vesical pouch from the rectum in cases of ruptured bladder was suggested by Dr. Harrison in his well-known paper in the 'Dublin Journal of Medical Science,' 1836, but I am not aware of its having been performed.

Although post-mortem the lower part of the tear in the bladder was found open from the slipping of the knot or giving way of the catgut, I think it was completely closed at the operation, since clear urine in large quantity flowed through the catheter up to the fifth day, when bloody urine was noted in the evening, and the whole of the urine passed on the last day contained blood. I may say, that the first stitch was put in at the lower end of the opening by means of a needle set at right angles to the handle, and was then firmly tied. One end of the catgut being then used by an assistant to pull the bladder up out of the pelvis, I threaded the other end into an ordinary needle, and carefully sewed the opening up with a continuous suture, a great part of which is still visible in the preparation.

Putting aside the few undoubted cases of recovery from rupture of the bladder outside the peritoneum, we come to the cases of alleged recovery after rupture into the peritoneum, about which much controversy has arisen, and mainly because those surgeons who regard rupture of the bladder as a necessarily fatal accident, refuse to believe in a recovery without a demonstration of the lesion, which is of course impracticable. A careful consideration of $\mathrm{Mr}$. Chaldecott's case, ' Provincial Medical and Surgical Journal, 1846,' leaves no doubt in my mind that it was one of rupture into the peritoneum, and Mr. Aston Key seems to have fully endorsed Mr. Chaldecott's view. The patient was treated by catheterism and opium, and suffered from peri- 
tonitis, and gout presumably due to absorption of urine from the peritoneal cavity. It is of course possible that the urine may have been shut off by adhesions from the general cavity of the peritoneum, but it is remarkable that on the sixth day after the accident the patient having attempted to micturate " felt something give way, and a burning pain all over his stomach and bowels, as if boiling water had been poured over them, and the same symptoms of faintness and distress as when the accident first happened."

Dr. Gross, of Philadelphia, in his work entitled, "Practical Treatise on the Diseases and Injuries of the Urinary Bladder," \&c., published in 1851, proposed abdominal section for the evacuation of the urine in these cases, and Dr. Walter has reported in the 'Philadelphia Medical and Surgical Reporter,' February, 1862 (quoted in Ranking's 'Abstract,' 1862, vol. ii), the case of a man, aged 26, in whom, ten hours after the accident, an incision was made in the linea alba, and a sponge introduced into the peritoneal cavity. A pint of urine and blood was thus mopped out, and a rent two inches long was observed in the base of the bladder. The bladder was left to itself and the catheter used, but apparently not tied in. The patient made a complete recovery.

It should be mentioned, that both Bonet ${ }^{1}$ and Cusack ${ }^{2}$ had performed Paracentesis abdominis for the evacuation of urine, but with fatal results.

In the 'Dublin Quarterly Journal of Medical Science' for November, 1868, Dr. Henley Thorp reported, perhaps the most remarkable case of recovery after ruptured bladder on record. A farmer, aged 30, was thrown from his horse and was found insensible. When he became conscious "he experienced a severe pain at the bottom of his belly, attended with an urgent desire to pass water, but no power of emptying his bladder." Dr. Thorp found him sitting up in great distress, and passed a full-sized gum elastic catheter into the bladder. "At first no fluid escaped; but upon

\footnotetext{
1 'Sepulchretum Anat.' lib. iii, sec. xxiv, obs. 12.

2 'Dublin Hosp. Reports,' 1818, vol, ii, p. 312.
} 
pushing the instrument onwards, and at the same time turning it a little on its axis, about a tablespoonful of bloody urine flowed out. By changing the position of the patient from side to side, turning him over upon his knees, and substituting a silver for the gum elastic instrument, I at length succeeded (says Dr. Thorp) in obtaining nearly half a pint of urine mixed with blood." A few hours later (it is not stated how many after the accident) Dr. Thorp passed an elastic catheter into the bladder, when "its movements were at first restricted and painful, until after cautiously probing and turning its point, it entered nearly its full length, when a different feeling of resistance was communicated, and it could be moved about with somewhat greater freedom." A tablespoonful of reddish urine now escaped, and Dr. Thorp proceeded to inject warm water from a half-pint elastic bottle three several times, allowing each eight ounces to be retained for a couple of minutes, and then to return through the catheter. "At first the water returned of a reddish tinge, but the last half-pint was clear and bloodless." The catheter was now withdrawn partially, so that its end projected into the bladder, and when the urine began to come away was tied in. Forty leeches were applied to the abdomen followed by poultices, and 1 grain of opium with $\frac{1}{2}$ grain of calomel was given every two hours. The catheter was kept in for eleven days and the patient recovered, having had symptoms of sub-acute peritonitis more than once.

In 1863 Dr. Stephen Smith, of New York, suggested cutting into the bladder from the perinæum, as in lateral lithotomy, in order to give free exit to the urine in cases of ruptured bladder. The operation has apparently been done only twice, viz. by Dr. W. J. Walker, in a case of laceration of the front of the bladder outside the peritoneum; and secondly, by Dr. Erskine Mason ('New York Medical Journal,' 1872) in a case of presumed rupture into the peritoneum. Both patients recovered.

In Dr. Mason's case the operation was done two days after the accident, when symptoms of general peritonitis 
had set in, and per rectum a tumour could be felt posterior to the prostate and to the left side. The bladder having been opened on a staff in the usual way, Dr. Mason felt confident that his finger detected a rent in the posterior wall of the bladder, but he did not examine the opening thoroughly, fearing to do injury. A quantity of bloody urine escaped through the incision, and the tumour felt per rectum-presumably the recto-vesical pouch distended with fluid-disappeared. In a fortnight the patient was convalescent and completely recovered.

Mr. Willett, who quotes Dr. Mason's case at some length, controverts his view as to the bladder having been ruptured into the peritoneum, though he allows that the operation saved the man's life. I am not aware that the operation has been performed in a similar case in this country; but $\mathrm{Mr}$. Bryant, in his ' Manual of Surgery,' recommends the proceeding in cases of ruptured bladder.

To sum up the cases of recovery after reputed rupture into the peritoneum, they are four in number:

1. Mr. Chaldecott's case, treated by catheterism of the bladder and peritoneum.

2. Dr. Walter's case, treated by abdominal incision to evacuate the urine.

3. Dr. Thorp's case, treated by catheterism of the bladder and peritoneum, and washing out the peritoneum through the catheter.

4. Dr. Mason's case, treated by lateral lithotomy.

These cases alone are sufficient to encourage the surgeon not to abandon a case of ruptured bladder as utterly hopeless, and Dr. Thorp's seems to me the most satisfactory of them all. An abdominal section for mere evacuation of fluid seems to possess little advantage over paracentesis abdominis in the linea alba, and must be less effective as a drain than tapping the recto-vesical pouch per rectum. Having experienced the very great difficulties to be encountered in stitching up the bladder with plenty of able assistants, I cannot recommend the proceeding for adoption when there would be the best chance of success, viz. imme-

vOL. LXII. 
diately after the accident when the surgeon first sees his patient. I may say that I was well acquainted with Dr. Thorp's case, but was not aware of Mr. Willett's experience. Had I known that the trial of stitching up the bladder had been made without success, I believe I should have resorted to Dr. Thorp's expedient of washing out the peritoneum, which I would certainly recommend for imitation in future cases. It seems to me to offer as good, if not better chances of success than any other proceeding, and has the great advantage that it can be put in action promptly, which is after all the great point. In addition it introduces no new element of danger to the patient, nor any serious surgical proceeding which may be distasteful to his friends. The operation of lateral lithotomy might, I think, be fairly reserved for cases of ruptured bladder in which the catheter could not be passed into the peritoneum from the urethra. 